\title{
S-carboxymethylcysteine in the treatment of glue ear: quantitative systematic review Robert Andrew Moore ${ }^{* 1}$, Dermot Commins ${ }^{2}$, Grant Bates ${ }^{2}$ and Ceri J Phillips ${ }^{3}$
}

Address: ${ }^{1}$ Pain Research and Nuffield Department of Anaesthetics, University of Oxford, Oxford Radcliffe Hospitals, The Churchill Headington, Oxford, OX 3 7LJ, UK, ${ }^{2}$ ENT Department, Radcliffe Infirmary, Oxford, OX2 6HE, UK and ${ }^{3}$ School of Health Sciences, University of Wales, Swansea Singleton Park, Swansea, SA2 8PP, UK

E-mail: Robert Andrew Moore* - andrew.moore@pru.ox.ac.uk; Dermot Commins - dcommins@freeuk.net; Grant Bates - grant.bates@surgery.oxford.ac.uk; Ceri J Phillips - C.J.Phillips@swansea.ac.uk

${ }^{*}$ Corresponding author

This article is available from: http://www.biomedcentral.com/I47I-2296/2/3

(C) 200 I Moore et al; licensee BioMed Central Ltd. Verbatim copying and redistribution of this article are permitted in any medium for any non-commercial purpose, provided this notice is preserved along with the article's original URL. For commercial use, contact info@biomedcentral.com

\begin{abstract}
Objective: To establish the clinical relevance of S-carboxymethylcysteine in the treatment of glue ear in children using measures approximating those saving a child from operation for grommet insertion.

Data sources: Cochrane Library, MEDLINE, EMBASE, PubMed, reference lists and reviews were used for randomised controlled trials comparing S-carboxymethylcysteine with placebo. Seven trials involving 283 children and I 46 ears were found.
\end{abstract}

Review methods: Studies were randomised, double-blind comparisons of Scarboxymethylcysteine (any dose and duration) with placebo in otitis media with effusion. Quality of trial reporting and validity of methods were assessed and used in sensitivity analysis. Main outcomes were relative benefit and number-needed-to-treat to prevent one grommet operation compared with placebo.

Results: Successful outcomes were obtained in 17\% of children given placebo (range $5 \%$ to $38 \%$ in individual studies) and in 35\% of children given S-carboxymethylcysteine (range 22 to $80 \%$ ). For combined data (children and ears) the relative benefit was $2.0(95 \% \mathrm{Cl} 1.4$ to 2.8$)$ and numberneeded-to-treat 5.5 ( $95 \%$ confidence interval 3.8 to 9.8). Pooled data from trials of higher reporting quality (4/7) or methodological validity (3/7) tended to have lower efficacy but were not statistically different from those of lower quality or validity.

Conclusion: S-carboxymethylcysteine is effective in the treatment of children with glue ear. For every five or six children treated with S-carboxymethylcysteine over one to three months, one will not undergo surgery for grommet insertion who would have done had they been given placebo. The confidence in this conclusion is limited because studies included relatively few children. 


\section{Background}

Glue ear is a common cause of hearing impairment in children, and was reviewed in an Effective Health Care Bulletin in 1992 [1]. It can result in a hearing loss (measured in decibels of hearing loss, dB HL) of o to $50 \mathrm{~dB} \mathrm{HL}$, with an average of $20 \mathrm{~dB} \mathrm{HL}$. This degree of hearing loss can turn normal speech into a whisper. Unilateral glue ear is less of a problem, but bilateral glue ear with significant hearing loss is commonly considered to pose developmental problems to children. Compared with watchful waiting, early surgery to introduce ventilation tubes significantly reduced behavioral problems nine months later [2]. Persistent bilateral hearing impairment of 25-30 dB HL is thought sufficient to justify surgery.

The epidemiology of glue ear has been extensively reviewed [1]. It is estimated that about $6 \%$ of two-year-olds have bilateral hearing impairment of at least $25 \mathrm{~dB} H \mathrm{HL}$ that persists for at least three months. Persistent bilateral glue ear of at least three months has a natural resolution rate of only $15 \%$ over 18 months [2] and 20-30\% over two years $[3,4]$.

The average rate of surgical treatment for glue ear in England is about 5/100o children under 15 years, and it is the most common operation in children, with as many as 60,000 operations annually [4].

An overview [6] concluded that S-carboxymethylcysteine increased the resolution rate significantly. That review did not take into account methodological quality of trials, and the outcomes chosen were unclear. Moreover, the statistical results could not be confirmed by recalculating the numbers given. The aim of this review was to re-search the literature, add at least one new study, and apply methodological quality filters in a sensitivity analysis to test the strength of the conclusion that mucolytics are beneficial in watchful waiting for glue ear.

An additional aim was to generate not only a statistical output (whether S-carboxymethylcysteine was better than placebo), but also to try to establish how much better it was. This might be described as the therapeutic effort, measured by the number needed to treat (NNT). NNT is treatment specific. It describes the difference between active treatment and control in achieving a particular clinical outcome [7]. Low NNTs indicate high treatment-specific efficacy. An NNT of 1 says that a favourable outcome occurs in every patient given the treatment but in no patient in a comparator group. This would be the 'perfect' result in, say, a therapeutic trial of an antibiotic compared with placebo with a sensitive organism. NNTs of 2 to 5 are indicative of high efficacy (as, for instance, with analgesics in acute pain [8]).

\section{Methods}

We sought randomised comparisons of S-carboxymethylcysteine with placebo in children with otitis media with effusion. A number of databases, including the Cochrane Library (issue 2, 2001), MEDLINE (1996-March 2001), EMBASE (to August 2000) and PubMed (to June 2001) were searched using "S-carboxymethylcysteine", "Carbocysteine", "S-CMC" and "mucodyne" as free text terms, and abstracts were then examined to see whether they had any prospect of qualifying. If they did, copies of the papers (any language) were obtained and read, and translated if necessary; the fate of all such papers is reported. The database of a pharmaceutical company (Rhone-Poulenc Rorer) was also examined for any published or unpublished studies. Reference lists of papers obtained and a previous overview [6] were also examined.

To be included, studies had to have been randomised comparisons of S-carboxymethylcysteine (any dose and duration) in otitis media with effusion in children aged under 16 years, to be full published papers, and to have a placebo comparison group. Abstracts were not considered. Authors were not contacted.

We were interested in outcomes (including adverse effects) for children, or failing that for individual ears, which could be interpreted as saving a child from an operation for grommet insertion. Ideally this would be a tympanogram type B becoming type A. Where this was not available (because several studies were quite old) we chose outcomes which would today influence the making of the clinical decision not to operate. Definitions used were:

- A normal tympanogram

- Reversion to normal audiometry

- Normal otoscopic findings

- Parental view (improvement/lack of problem).

For instance, in the earliest report [9] objective assessment was based on four factors - hearing levels for sound and speech, tuning fork tests, the appearance of the tympanic membranes and pure tone audiology. At the final assessment children could have an outcome that was excellent (a normal history with no more than 10 decibels hearing loss at any frequency), improved (subjective improvement and improvement in one of the four objective criteria or improvement in three of the four objective factors), unchanged, or worse. Our judgement was that the top two categories constituted sufficient improvement to avoid surgery today. Fourteen of the 16 
successes with carboxymethylcysteine and one of the three with placebo in this trial [9] were in the first of these categories. Similar judgements based on criteria used today to decide about surgery were made for the other papers. Contemporary practice is that children should have a history of deafness proven by audiology and a type B tympanogram, and should have undergone a period of watchful waiting before surgery to ensure that spontaneous resolution was unlikely.

Each report which could possibly be described as a randomised controlled trial was read independently by at least three authors (RAM, DC, GB) and scored using a commonly-used three item, 1-5 score, quality scale. [10] Consensus was then achieved. The maximum score of an included study was 5 and the minimum score was 2 . Authors were not blinded because they already knew the literature. We also used a scale assessing trial validity (DC, GB)[11], in which the maximum score was 16. Where appropriate we adjusted the validity scale [11] to reflect current practice and to ensure clinical homogeneity. Analysis was by intention-to-treat where possible. For some studies which included children whose disease description would not be adequate for grommet insertion today, we extracted data only on those children where there was sufficient information to judge that an operation would be likely today (additional file 1 ).

Confidence intervals (95\%) for single samples were calculated for proportions [12]. Relative benefit and relative risk estimates were calculated with $95 \%$ confidence intervals using a fixed effects model [13]. Heterogeneity tests were not used as they have previously been shown to be unhelpful [14], though homogeneity was examined visually [15]. Publication bias was not assessed using funnel plots as these tests have been shown to be unhelpful $[16,17]$. The number needed to treat (NNT) with confidence intervals was calculated by the method of Cook and Sackett [18] from the sum of all events and patients for treatment and placebo.

Relative benefit or risk was considered to be statistically significant when the 95\% confidence interval did not include 1. NNT or NNH values were only calculated when the relative risk or benefit was statistically significant, and are reported with the 95\% confidence interval. Statistical significance of any difference between numbers needed to treat was assumed if there was no overlap of the confidence intervals. Calculations were performed using Microsoft Excel 98 on a Power Macintosh G4.

\section{Results}

Twenty five reports were found and considered for this review. Nineteen studies were excluded for various reasons (additional file 2). Four were animal experiments, six were not randomised (including a study conducted post myringotomy), two were on postoperative children, four were letters with no data, two had no extractable data and one was a retrospective study. Seven published randomised, double-blind, placebo-controlled trials met the inclusion criteria. Four studies [19-22] had information on affected children, while three $[9,23,24]$ had information on affected ears. One study [9] included one adult and 45 children. Details of the seven included studies, together with entry criteria, dose and duration of treatment, outcomes and quality and validity scores are shown in (additional file 1).

Information was available for 283 children and 146 ears. S-carboxymethylcysteine was given as a syrup or linctus at various doses (sometimes age-related) for periods as little as 10 or 15 days [22,24], but other studies used it for between one and three months. Only two studies [9,22] individually showed statistical benefit of treatment over placebo (Figure 1). (additional file 3) has the number of events, relative benefits and NNT for each individual trial.

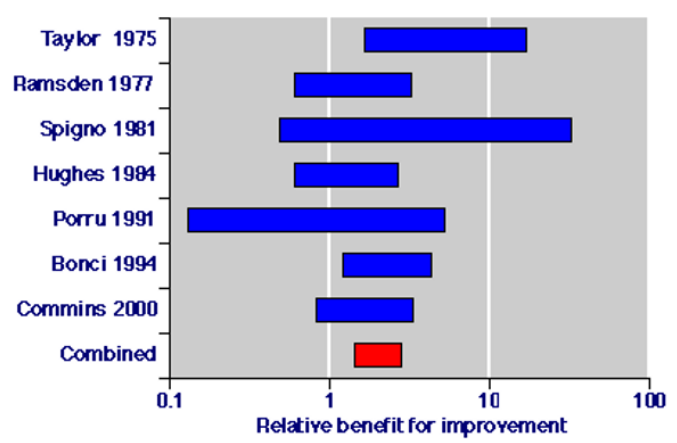

Figure I

Relative benefit calculated for each individual trial (blue) and the combined data (red). The bars represent the limits of the $95 \%$ confidence interval of the relative benefit. Where this crosses I, this indicates no statistically significant difference from placebo.

The L'Abbé plot for individual studies is shown in Figure 2 . The only study below the line of equality [24] had extractable outcomes for Type B to Type A tympanograms available for only three children in the placebo group. We included this trial in order to be inclusive, and because it would have a small weight in the overall results.

Successful outcomes were obtained in $17 \%$ of children given placebo (range $5 \%$ to $38 \%$ in individual studies) 


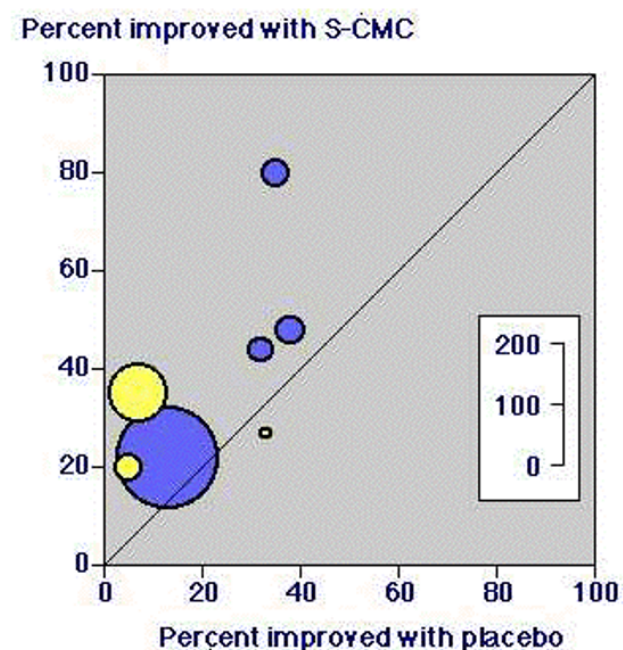

Figure 2

Improvement with S-carboxymethylcysteine or placebo in individual trials Each trial is represented by a symbol, the size of which is proportional to the size of the trial (inset). Those in blue represent results for children, and those in yellow results for ears. Symbols above the line of equality indicate where there was a trend towards S-carboxymethylcysteine being more effective than placebo.

and in $35 \%$ of children given S-carboxymethylcysteine (range 22 to $80 \%$ ). The numbers-needed-to-treat for children, ears, combined data and sensitivity analyses are shown in Table 1 . All analyses showed statistical homogeneity, despite the low power of conventional tests to demonstrate this [14]. There was significant treatment effect, with similar magnitudes of effects for both children and ears. The combined data had a number-needed-to-treat of 5.5 (95\% confidence interval 3.8 to 9.8). This means that for every five or six children treated with S-carboxymethylcysteine over one to three months, one will not undergo surgery for grommet insertion who would have done had they been given placebo.

Four studies had quality scores of 3 or more $[9,19,20,24]$. The number-needed-to-treat for these high quality studies was 6.7 (4.2 to 17). Three studies [19-21] had validity scores of 8 or more. These failed to reach statistical significance with a relative benefit of 1.5 (o.96 to 2.3) and a number-needed-to-treat of 8.5 (4.4 to 105). For four studies $[9,19,22,23]$ intention-to-treat analysis was possible. The number-needed-to-treat for these studies was 5.1 (3.9 to 8.3); there was no statistical benefit in the three trials in which only per protocol data could be used.
Two trials $[9,19]$ had more than 50 patients in the comparison, and in these the NNT was 6.3 (4.0 to 15). Confidence intervals of numbers-needed-to-treat of large and small trials, of trials of high and low quality, of high and low validity, and with intention-to-treat or per protocol analysis overlapped (Table 1).

Definite comments about adverse effects were reported in only three studies. $[9,19,22]$ Only one adverse effect was recorded in a child given S-carboxymethylcysteine who had a rash and vomited.

\section{Discussion}

Glue ear in children is a significant cause or morbidity, in which hearing loss may affect development. Because glue ear resolves spontaneously in most children, watchful waiting has been recommended for up to six months before surgery [1], and this recommendation is now common practice. Variability in the success rates with placebo between $5 \%$ and $38 \%$ in these seven studies is likely to reflect different policies over a quarter century, as well as the random play of chance.

On average (Table 1) for every 100 children given placebo in the seven trials, 17 would have avoided surgery (95\% confidence interval 11 to 23). That figure is similar to rates in contemporary British settings. In Dundee in the late 1980s, 33 of 191 (17\%) of children referred for surgery resolved without surgical treatment [25]. In the placebo group of the large randomised study in Oxford in the late 1990 , the resolution rate without surgery was $13 \%$ [19], while in a watchful waiting arm of another large randomised study in Bristol $15 \%$ did not require surgery [2]. In terms of resolution rates, the pooled data in the meta-analysis resembled clinical practice.

Children who have not undergone a period of watchful waiting may be regarded as having a less severe form of the disease, and S-carboxymethylcysteine may be less effective where the disease was longer established. In the four trials in this meta-analysis with placebo resolution rates of $32-38 \%$ the number of children was small, at 3 , 16,19 and 20 respectively, contributing about a quarter of those given placebo. Disease severity is unlikely to confound the results, therefore.

On average (Table 1) for every 100 children given S-carboxymethylcysteine in the seven trials, 35 would have avoided surgery (95\% confidence interval 29 to 41 ). There was a consistent benefit for use of S-carboxymethylcysteine with an overall relative benefit of 2.0 (1.4 to 2.8 ). One child in every five or six given S-carboxymethylcysteine for one to three months would be spared an operation for grommet insertion. Only one adverse effect was reported in these seven trials. 
Table I: Summary of results

\begin{tabular}{|c|c|c|c|c|c|}
\hline \multirow[b]{2}{*}{ Results by: } & \multirow[b]{2}{*}{ Number of studies } & \multicolumn{2}{|c|}{ Number/Total (\%) improved } & \multirow[b]{2}{*}{$\begin{array}{c}\text { Relative benefit } \\
(95 \% \mathrm{CI})\end{array}$} & \multirow[b]{2}{*}{ NNT $(95 \% \mathrm{Cl})$} \\
\hline & & with S-CMC & with placebo & & \\
\hline Children & 4 & $\begin{array}{c}54 / 14 \\
(38)\end{array}$ & $\begin{array}{c}30 / 140 \\
(21)\end{array}$ & $1.7(1.2$ to 2.4$)$ & 6.1 (3.7 to 17) \\
\hline Ears & 3 & $\begin{array}{c}23 / 77 \\
(30)\end{array}$ & $\begin{array}{c}5 / 69 \\
(7)\end{array}$ & $3.8(1.6$ to 9.1$)$ & 4.4 (2.9 to 9.3$)$ \\
\hline Combined & 7 & $\begin{array}{c}77 / 220 \\
(35)\end{array}$ & $\begin{array}{c}35 / 209 \\
(17)\end{array}$ & $2.0(1.4$ to 2.8$)$ & 5.5 (3.8 to 9.8$)$ \\
\hline \multicolumn{6}{|c|}{ Sensitivity analysis (ears or children) } \\
\hline $\begin{array}{l}\text { Quality score } \\
\geq 3\end{array}$ & 4 & $44 / 153$ & $21 / 153$ & 2.1 ( 1.3 to 3.3$)$ & 6.7 (4.2 to I7) \\
\hline $\begin{array}{l}\text { Quality score } \\
\leq 2\end{array}$ & 3 & $33 / 67$ & $14 / 56$ & $1.9(1.2$ to 3.1$)$ & 4.1 (2.5 to 13) \\
\hline $\begin{array}{l}\text { Validity score } \\
>8\end{array}$ & 3 & $38 / 123$ & $23 / 120$ & $1.5(0.96$ to 2.3$)$ & 8.5 (4.4 to 105$)$ \\
\hline $\begin{array}{l}\text { Validity score } \\
\leq 7\end{array}$ & 4 & $39 / 97$ & $12 / 89$ & $3.0(1.7$ to 5.1$)$ & 3.7 (2.6 to 6.8$)$ \\
\hline $\begin{array}{l}\text { Intention-to- } \\
\text { treat }\end{array}$ & 4 & $53 / 164$ & $22 / 17 \mid$ & 2.5 (1.6 to 3.9$)$ & 5.1 (3.6 to 9.3$)$ \\
\hline Per protocol & 3 & $24 / 56$ & $13 / 38$ & 1.3 (0.75 to 2.2$)$ & $11.6(3.5$ to -8.9$)$ \\
\hline $\begin{array}{l}\text { More than } 50 \\
\text { in } \\
\text { total }\end{array}$ & 2 & $33 / 124$ & $|4 / 13|$ & $2.5(1.4$ to 4.5$)$ & $6.3(4.0$ to 15$)$ \\
\hline $\begin{array}{l}\text { Fewer than } \\
50 \\
\text { total }\end{array}$ & 5 & $44 / 96$ & $21 / 78$ & 1.7 (1.2 to 2.6$)$ & 5.3 (3.0 to 20$)$ \\
\hline
\end{tabular}

How confident can we be of this result? Clearly there is the potential for negative studies not to have been published, though no unpublished studies were found in searching a manufacturer's database of trials. Extensive searching both here and in a previous review [6] using a different search strategy found the same studies. We excluded one study [26] from the previous review because it included children having myringotomy. Quality scores were 2 in three studies and 4 or 5 in four. We know that a quality score of 2 or less may be associated with overestimation of treatment effects $[27,28]$. Here, both lower quality scores, lower validity scores, and trials with fewer than 50 children yielded non-significantly better estimates of effects (Table 1). Intention to treat analysis produced better results than per protocol analysis, in that per protocol analysis was not significantly different from placebo in three small studies.
Sensitivity analysis showed that choosing only studies of higher quality, higher validity, those that reported intention to treat results, or were larger made no appreciable difference to the overall conclusion. The proviso, though, is that for these sensitivity analyses the numbers of children or ears was small [29], and no definitive conclusions should be drawn other than the absence of gross effects.

Outcomes chosen by investigators over 25 years differed. We chose to use endpoints that would today be reliable indicators that a child could sensibly be spared an operation for grommet insertion. Re-analysis of the Pignatoro review [6] was necessary because it did not choose sparing operation as an outcome, because we were unable to reconcile the data in that paper to the odds ratios given, because we wanted outputs in the form of number-needed-to-treat, and to add information from our own study [19]. 
When treatments have small effects and studies have small numbers, a clinically significant effect of treatment may be missed [29]. To be sure of the direction and the magnitude of treatment large numbers of children have to be studied, and for S-carboxymethylcysteine studies of over 1000 children would have to be conducted to be sure of the number-needed-to-treat with a low confidence interval [29]. This review included fewer than 300 children and 150 ears, reflecting the wide confidence interval of the number-needed-to-treat estimate.

Despite this, S-carboxymethylcysteine is likely to be a useful treatment of children during a period of watchful waiting before surgery. It spares about $18 \%$ of children an operation, and as well as being clinically effective an economic analysis [30] showed it to be cost-effective when the number-needed-to-treat is better (lower) than 15 . With a number-needed-to-treat of about 5 , use of Scarboxymethylcysteine would probably result in fewer children having surgery for grommet insertion, and actually reduce overall costs as well as relieving pressure on surgery time and waiting lists.

However, if spontaneous resolution rates were much lower than $10 \%$ any conclusion about cost-effectiveness might have to be reviewed. If the relative benefit of 2 found in this meta-analysis was applied to different spontaneous resolution rates, different numbers needed to treat would be produced. With a $20 \%$ spontaneous resolution rate doubled to $40 \%$ with S-carboxymethylcysteine, the NNT would be 4 . Doubling a $15 \%$ rate to $30 \%$ gives an NNT of 7 . Doubling a $10 \%$ rate to $20 \%$ gives an NNT of 10 , but doubling a $5 \%$ rate to $10 \%$ gives an NNT of 20. This demonstrates that statistical outputs, while crucial to any analysis, may be less important than therapeutic effort in making clinical policy decisions.

One strength of this review came from choosing trials and patients that would today be likely to have surgery for grommet insertion because of the severity of their disease, and demonstrated unequivocally using tympanography and audiometry, and after watchful waiting. It ensured clinical homogeneity. Another strength was the choice of outcome to ensure that an operation would not be carried out because of disease resolution, again using contemporary criteria. The weakness is that despite having access to data from seven studies, the total numbers are small, and the total numbers available for sensitivity analysis makes that unreliable.

\section{Conclusions}

There is a clear indication for a large randomised trial carried out in real-world circumstances.

\section{Competing interests}

RAM and CJP have been paid consultants for RhonePoulenc Rorer. Rhone-Poulenc Rorer also made a financial contribution to a randomised trial [19] carried out in Oxford.

\section{Additional material}

\section{Additional file 1}

An Excel table describing the studies included in the analysis Click here for file

[http://www.biomedcentral.com/content/supplementary/14712296-2-3-S1.xIs]

\section{Additional file 2}

An Excel table giving excluded reports and reasons for their exclusion

Click here for file

[http://www.biomedcentral.com/content/supplementary/14712296-2-3-S2.xIs]

\section{Additional file 3}

Outcomes used to calculate results, plus the results for individual studies

Click here for file

[http://www.biomedcentral.com/content/supplementary/14712296-2-3-S3.xIs]

\section{Acknowledgements}

Support was obtained from Anglia \& Oxford NHSE R\&D Directorate, Rhone-Poulenc Rorer, and Pain Research funds. Helena Fingerová kindly translated Czech papers, and Rhone-Poulenc Rorer obtained translations of papers in Japanese and Italian.

\section{References}

I. Anon: The treatment of persistent glue ear in children. Effective Health Care, 1992, Number 4:

2. Wilks J, Maw R, Peters TJ, Harvey I, Golding J: Randomised controlled trial of early surgery versus watchful waiting for glue ear: the effect on behavioural problems in pre-school children. Clin Otolaryngol 2000, 25:209-2 I4

3. Maw AR, Parker A: Surgery of the tonsils and adenoids in relation to secretory otitis media in children. Acta Otolaryngol 1998. Supp 454:202-207

4. Lieberman A, Bartal N: Untreated persistent middle ear effusion. J Laryngol Otolaryngol 1986, 100:875

5. Isaacson G, Rosenfeld RM: Care of the child with tympanostomy tubes. Ped Clin N America 1996, 43:1 I83-I I 93

6. Pignatoro O, Pignatoro LD, Gallus G, Calori G, Cordano Cl: Otitis media with effusion and S-carboxymethylcysteine and/or its lysine salt: a critical overview. Int J Ped Otorhinolaryngol 1996, 35:23I-24I

7. McQuay HJ, Moore RA: Using numerical results from systematic reviews in clinical practice. Ann Intern Med 1997, I 26:712720

8. McQuay HJ, Moore RA: An evidence-based resource for pain relief. Oxford. Oxford University Press 1998, ISBN 0-19-2627 I 8-X:

9. Taylor PH, Dareshani N: S-Carboxy-methyl-cysteine syrup in secretory otitis media. Br J Clin Pract 1975, 29:177-180

10. Jadad AR, Moore RA, Carroll D, Jenkinson C, Reynolds DJM, Gavaghan DJ, McQuay HJ: Assessing the quality of reports of randomized clinical trials: is blinding necessary? Control Clin Trial 1996, 17:1-12 
II. Smith LA, Oldman AD, McQuay HJ, Moore RA: Teasing apart quality and validity in systematic reviews: an example from acupuncture trials in chronic neck and back pain. Pain, 2000, 86:119-132

12. Gardner MJ, Altman DG: Confidence intervals rather than $\mathbf{P}$ values: estimation rather than hypothesis testing. BMJ 1986, 292:746-50

13. Morris JA, Gardner MJ: Calculating confidence intervals for relative risk, odds ratios and standardised ratios and rates. In Statistics with confidence - confidence intervals and statistical guidelines. Edited by Gardner, MJ and Altman DG. London: British Medical Journal, 199550-63

14. Gavaghan DJ, Moore RA, McQuay HJ: An evaluation of homogeneity tests in meta-analyses in pain using simulations of individual patient data. Pain 2000, 85:415-424

15. L'Abbé KA, Detsky AS, O'Rourke K: meta-analysis in clinical research. Ann Intern Med 1987, 107:224-233

16. J Tang, JL Liu: Misleading funnel plot for detection of bias in meta-analysis. J Clin Epidemiol 2000, 53:477-484

17. Sterne JA, Gavaghan D, Egger M: Publication and related bias in meta-analysis. Power of statistical tests and prevalence in the literature . J Clin Epidemiol 2000, 53: II I 9-29

18. Cook RJ, Sackett DL: The number needed to treat: a clinically useful measure of treatment effect. BM] 1995, 3 I 0:452-4

19. Commins DJ, Koay BC, Bates GJB, Moore RA, Sleeman K, Mitchell B, Bates $S$ : The role of Mucodyne in reducing the need for surgery in patients with persistent otitis media with effusion. Clin Otolaryngol 2000, 25:274-9

20. Ramsden RT, Moffat DA, Gibson WP, Jay MM: S-carboxymethylcysteine in the treatment of glue ear.J Laryngol Otol 1977, 9:847851

2I. Hughes KB: Management of middle-ear effusions in children. J Laryngol Otol 1984, 98:677-684

22. Bonci M, Bozzi A: Terapia mucoregulatrice nella patalogia secernente dell'orecchio medio. Minerva Medica 1994, 85:83-7

23. Spigno G, Teatini GP: Assessment of the effectiveness of carbocysteine in the treatment of catarrhal otitis. Otorinolaringologia | 98I, 3 I:I-4

24. Porru P, Sulis G, Setzu : Valuatione dell'efficacia e tollerabilita di S-carbossimetilcisteina sale de lisina nel trattamento sella otite media catarrale a timpano chiuso in età pediatrica. Orl Ped |99|, II:249-254

25. Mills R: Risk factors for chronicity in childhood otitis media with effusion. Clin Otolaryngol 1999, 24:343-345

26. Kumazawa T, Ushiro K: Clinical evaluation of S-CMC syrup applied in the treatment of otitis media with effusion. Acta Otolaryngol 1988, Supp 458:56-62

27. Khan KS, Daya S, Jadad AR: The importance of quality of primary studies in producing unbiased systematic reviews. Arch Intern Med 1996, 1 56:66I-6

28. Moher D, Pham B, Jones A, et al: Does quality of reports of randomised trials affect estimates of intervention efficacy reported in meta-analyses? Lancet 1998, 352:609-613

29. Moore RA, Gavaghan D, Tramèr MR, Collins SL, McQuay HJ: Size is everything - large amounts of information are needed to overcome random effects in estimating direction and magnitude of treatment effects. Pain 1998, 78:209-216

30. Phillips CJ, Burrell A, Moore RA, Bates GJ, Commins D: Mucodyne: the economics of preventing surgery for grommets. J Med Econ 1999, 2:167-176

\footnotetext{
Publish with BioMed Central and every scientist can read your work free of charge

"BioMedcentral will be the most significant development for disseminating the results of biomedical research in our lifetime."

Paul Nurse, Director-General, Imperial Cancer Research Fund

Publish with BMC and your research papers will be:

- available free of charge to the entire biomedical community

- peer reviewed and published immediately upon acceptance

- cited in PubMed and archived on PubMed Central

- yours - you keep the copyright 